\title{
Analysis of Flow Field and Pumping Performance for a Valveless Piezoelectric Pump with a Hemisphere-segment Group
}

\author{
Caiqi $\mathrm{Hu}^{1}$ - Jing $\mathrm{Ji}^{1}{ }^{*}$ * - Xiaoqi $\mathrm{Hu}^{2}$ - Jude $\mathrm{Liu}^{3}$ - Shengduo $\mathrm{Li}^{1}$ \\ ${ }^{1}$ Qingdao Agricultural University, College of Mechanical and Electrical Engineering, China \\ 2Lishui University, College of Engineering and Design, China \\ 3The Pennsylvania State University, Department of Agricultural and Biological Engineering, USA
}

The pumping and mixing performance of a valveless piezoelectric pump were proposed and studied. A hemisphere-segment group that was fixed in the pump played the role of a valve. Based on the theoretical analysis of the pumping performance, the changes of the fluid velocity field, pressure difference, the coefficient of resistance and flow rate were simulated with FLUENT. Simulation results revealed the relationships among the pressure field, flow rates, row and column numbers, and row and column intervals of the hemisphere-segment group. The simulation results indicated that increasing row and column numbers, increasing row intervals, and decreasing column intervals could all increase the flow resistance difference in pump flow field and thus increased the flow rate. It was also found that the pumping effect was significantly improved by increasing row number than increasing column number; the increasing of row and column intervals could increase the size and strength of the vortex, and then improve the mixing and stirring performance of this pump. Finally, simulation results were tested and verified through pump flow-rate experiments.

Keywords: hemisphere-segment group, array, valveless piezoelectric pump, simulation analysis, flow rate, mesh independence

Highlights

- $\quad$ The changing pattern of the pumping and mixing performance for a new valveless pump was proposed.

- The pumping performance was analysed based on the theory of hydrodynamics.

- The changes of the fluid velocity field, pressure differences, the coefficient of resistance and flow rates vs. HSG dimensions and arrangement were simulated.

- $\quad$ Simulation results were tested and verified through pump flow rate experiments.

\section{INTRODUCTION}

Traditional pumps use mechanical motion to create fluid flow. Unlike with the traditional pump, the principle of the valveless piezoelectric pump is to convert electrical energy into mechanical energy via the vibration of a piezoelectric vibrator. The resulting movement and force from this piezoelectric vibrator will then directly act on the fluid medium to create fluid flow [1]. Such a pump has no moving parts when controlling fluid in unidirectional flow. Thus, it has no mechanical wearing problems and lubrication requirements, as needed in traditional pumps [2]. Therefore, the valveless piezoelectric pump has advantages in low manufacturing costs [3], easy miniaturization, low energy consumption, and long service life, etc. [4]. It also has wide potential application in medical, health, mechanical, and electrical systems that require the miniaturization of mechanical components [5]. Moreover, the valveless piezoelectric pump has unique advantages, especially in the integrated piezoelectric mixing and conveying field for microflow liquid [6] and [7].
There are several types of no-moving-part valves with different structures for valveless piezoelectric pumps. Most previous studies were focused on valveless diffuser/nozzle-based fluid pumps [8]. For this type of pump, a pair of mutually inverted diffuser/ nozzle tubes was externally fitted to both sides of the pump chamber to form a one-way flow [9]. These two external tubes occupied some space and hindered the pump's further integration and miniaturization. A piezoelectric mixing pump was put forward by Rife et al. [10]. To form a homogeneous mixture, the piezoelectric vibrator in that pump drove the blocks in the mixing pool and created vortexes. This device could only mix and stir fluids, and was not able to convey them. Another piezoelectric mixing pump was reported by Sheen et al. [11], which integrated the functions of fluid mixing and pumping; but the block structures of this pump used for mixing were complex. A valveless piezoelectric pump with unsymmetrical slope elements were presented by Xia et al. [12], which could realize the integration of mixing and conveying functions. The unsymmetrical slope elements in the pump chamber bottom played the role of a no-moving-part valve. Meanwhile when 
the fluid flowed through unsymmetrical slopes, the vortexes and turbulence were formed, and the fluid was fully mixed and stirred. However, the unsymmetrical slopes increased the complexity of the pump structure and thus could cause difficulties in manufacturing. The valveless piezoelectric pump with hemisphere-segment (VPPHS) contains a hemisphere segment as a no-moving-part valve. The hemisphere segment (HS) is a $1 / 4$ sphere, which has features of a simple structure and good manufacturability. When the piezoelectric vibrator (PV) vibrates upward and downward in the pump chamber, the fluid is pumped because of flow resistance difference (FRD) between the round face and the spherical surface of HS [13]. For the valveless piezoelectric pump with a hemispheresegment group (VPPHSG), however, some HSs are arranged in a certain way to function as a nomoving-part valve. When the fluid flows through the hemisphere-segment group (HSG), the fluid and those hemisphere segments, interact with each other and generate turbulences and vortexes, which form a very complex flow field and create pressure differences. As a result, the turbulence/vortex make the fluid mixed or blended, and the pressure difference generates fluid flow. Thus, it is imperative to understand the interactions between HSG parameters and fluid flow and quantify their impacts on pump flow field and flow rate.

In this paper a VPPHSG, which can mix, stir and convey fluid, was proposed and studied. The objectives of this research were (1) to theoretically analyse the pumping process and flow rate; (2) to develop models based on different row and column numbers and intervals of HSs and use these models to calculate and simulate the velocity and pressure of the flow field, HSG resistance coefficient (RC), and the pump flow rate; (3) Then the effect of varying HSG parameters on pumping performance, as well as the influence of those parameters on mixing and stirring effect was analysed; (4) to verify the pump flow rate simulation results with experimental results.

\section{VALVE-LESS PUMP STRUCTURE}

The structure of VPPHSG is shown in Fig. 1. All HSs are arranged in a rectangle. All row intervals of HSs are equal, as are all column intervals. All HSs are oriented in the same way. The flat semi-circular surface of HS is perpendicular to the center connection line of Tubes $B$ and D. The spherical surface of HSs faces to the side of Tubes A, B and C. The diameters of Tubes A, $\mathrm{B}, \mathrm{C}$, and D are equal. The flat semi-circular surfaces of HSs face the side of tube D. Some dimensions of the pump used for simulation are listed in Table 1. When PV vibrates up and down in the pump chamber, the fluid is pumped in one way because of the FRD formed between the semi-circular plane and spherical surface of the HS [12]. Multiple HSs that are regularly arranged can undoubtedly amplify the FRD of a single HS and form a one-way flow from the side of Tubes $\mathrm{A}, \mathrm{B}$ and $\mathrm{C}$ to the side of Tube $\mathrm{D}$ in the pump chamber. Thus, the pumping performance could be better with HSG as a no-moving-part valve than with a single HS as a no-moving-part valve. Three different liquids in different proportions can be fed into the pump chamber through three tubes ( $\mathrm{A}, \mathrm{B}$ and $\mathrm{C})$. When different liquids flow through the complex flow field with HSG, these three liquids can be fully mixed and stirred because of turbulences and vortexes generated in the flow field. Mixed liquids are conveyed out through Tube D. The functions of mixing, stirring, and conveying are realized.

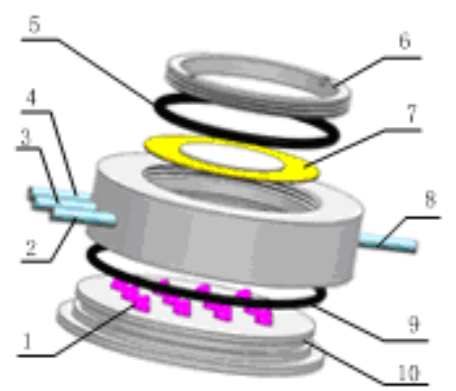

Fig. 1. Valveless pump structure; 1. HSG; 2. Tube A; 3. Tube B; 4. Tube C; 5. upper seal ring; 6. screw pump cover; 7. PV; 8. Tube D; 9. lower seal ring; 10. pump seat

To conduct theoretical analysis, when fluids are fed into the pumping chamber from Tubes A, B, and $\mathrm{C}$ and pumped out from tube $\mathrm{D}$, this flow direction is defined as forward flow (FF); conversely, if the fluid flows into the pumping chamber from Tube D and comes out from tubes A, B, and C, it is defined as reverse flow (RF). Tubes A, B, and $\mathrm{C}$ are defined as inlet tubes, and tube $\mathrm{D}$ is defined as an outlet tube.

\section{THEORETICAL ANALYSIS OF PUMPING}

The pumping performance of a piezoelectric pump depends on the characteristics of the no-movingpart valve [14]. For the VPPHSG, the total pressure loss when fluid flows through HSG with $m$ rows and $n$ column HSs is related to the flow resistance coefficient (FRC), flow velocity, and fluid density. Their relationships can be expressed as follows:

$$
\sum \Delta p_{F}=\sum\left(C_{D F}\right) \cdot \rho v_{F}^{2} / 2,
$$




$$
\sum \Delta p_{R}=\sum\left(C_{D R}\right) \cdot \rho v_{R}^{2} / 2
$$

where $\Sigma \Delta p_{F}$ and $\Sigma \Delta p_{R}$ are the total pressure loss of $\mathrm{FF}, \mathrm{RF}$, respectively, $\Sigma C_{D F}$ and $\Sigma C_{D R}$ are the total resistance coefficient of flow around HSG in FF, RF, respectively, $v_{F}$ and $v_{R}$ are the average velocity of FF, and RF, respectively, $\rho$ is fluid density.

The total pressure loss is the sum of all HSs pressure loss, and the total resistance coefficient is the sum of all RCs. It can be expressed as follows:

$$
\begin{aligned}
& \sum \Delta p_{F}=\sum_{i=1}^{m}\left(\sum_{j=1}^{n} \Delta p_{i j F}\right), \\
& \sum \Delta p_{R}=\sum_{i=1}^{m}\left(\sum_{j=1}^{n} \Delta p_{i j R}\right), \\
& \sum C_{D F}=\sum_{i=1}^{m}\left(\sum_{j=1}^{n} C_{D i j F}\right), \\
& \sum C_{D R}=\sum_{i=1}^{m}\left(\sum_{j=1}^{n} D_{D i j R}\right) .
\end{aligned}
$$

The flow quantity $Q$ of unit time for a pump can be expressed as follows [15] and [16]:

$$
Q=\Delta V f \frac{\sum C_{D R}-\sum C_{D F}}{2+\sum C_{D R}+\sum C_{D F}},
$$

where $\Delta V$ is the maximum variation of pump chamber volume in a vibration cycle of $\mathrm{PV}$, and $f$ is the vibration frequency of PV.

According to Eq. (7), when $\Sigma C_{D R}-\Sigma C_{D F} \neq 0$, $Q \neq 0$. This indicates that the one-way flow of fluid can be formed in the pump chamber. Because the HS has an asymmetric structure, and the resistances when fluid flowing around the spherical surfaces and the semi-circular plane of HS are unequal, the forward and reverse flow resistances of HSG are also unequal. This flow resistance difference makes the pump convey fluid in a single direction.

\section{FLOW FIELD ANALYSIS}

According to Eq. (7), when $\Delta V$ and $f$ are constant, $\Sigma C_{D R}$ and $\Sigma C_{D F}$ will have a great influence on the pump flow rate. The row and column numbers, row and column intervals of HSG have a direct influence on the pump flow rate and the resistance difference. The flow field is complex because of the resistance that HSG creates on the fluid in the pump chamber. The analytical solution for the variations of velocity and pressure could not be obtained. The simulation with FLUENT is an effective method to study the velocity and pressure in a flow field. In the following, the flow fields with different row and column numbers and different intervals of HSG are simulated and analysed to obtain the influence of those factors on the pumping performance.

\subsection{Numerical Mesh Analysis}

In this research, the models of a valveless pump with HSG arranged in a rectangle were established in FLUENT. The structural and geometric parameters are shown in Table 1 . The boundary conditions used for simulating the upward and downward cycle vibration of the piezoelectric vibrator was the sine function. The pressure at inlets and outlet was set at standard atmospheric pressure. The working medium of the pump was water, which was treated as a viscous incompressible Newtonian fluid.

This study included seven different models for simulating pump flow fields when the fluid flows through the hemisphere-segment group. To increase calculation efficiency, the unstructured mesh was found more suitable for this simulation. Therefore, it was used to construct the pump's internal flow field. Meanwhile, to receive more accurate flow field data, a smaller mesh and more elements per area or higher mesh density were used within the HSG and in those areas near the inlet and outlet of the pump.

Mesh independence was analysed based on different numbers of mesh elements. As an example, pump internal flow-field analysis results for a 3 by 4 HSG with fixed row interval $(S=12 \mathrm{~mm})$ and column interval $(H=12 \mathrm{~mm})$ are given in Fig. 2. This example included more than one different mesh densities or a different number of mesh elements.

The results of the simulation using the above models in Fig. 2 are given in Fig. 3. When increasing the number of mesh elements or mesh density, the

Table 1. Pump structure parameters for simulation

\begin{tabular}{ccccccc}
\hline \multirow{2}{*}{ Name } & \multirow{2}{*}{ Radius of HS } & Diameter of tube A & Diameter of PV & $\begin{array}{c}\text { Height of pump } \\
\text { chamber }\end{array}$ & \multicolumn{2}{c}{ Pump chamber } \\
\cline { 6 - 7 } Size $[\mathrm{mm}]$ & 4 & 4 & 50 & 6 & Outer diameter & Inner diameter \\
\hline
\end{tabular}


simulated flow rate decreases rapidly, and then tends to steady when the number of mesh elements is greater than 60,000 . When increasing mesh elements from 613,865 to $1,344,625$, the simulated flow rate was relatively constant. This is the evidence of mesh independence.

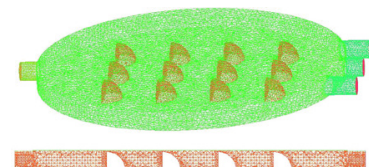

a)

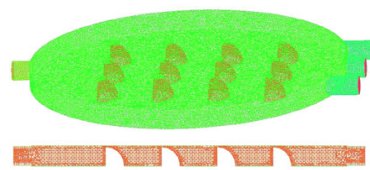

c)

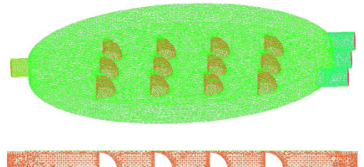

b)

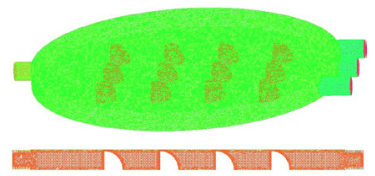

d)
Fig. 2. Mesh models using different densities of mesh elements; global mesh size is a) 0.4, b) 0.5 , c) 0.4 and d) 0.3 and there are a) 613,865 , b) 334,724 , c) 613,865 and d) 1,344,625 mesh elements in the pump flow field

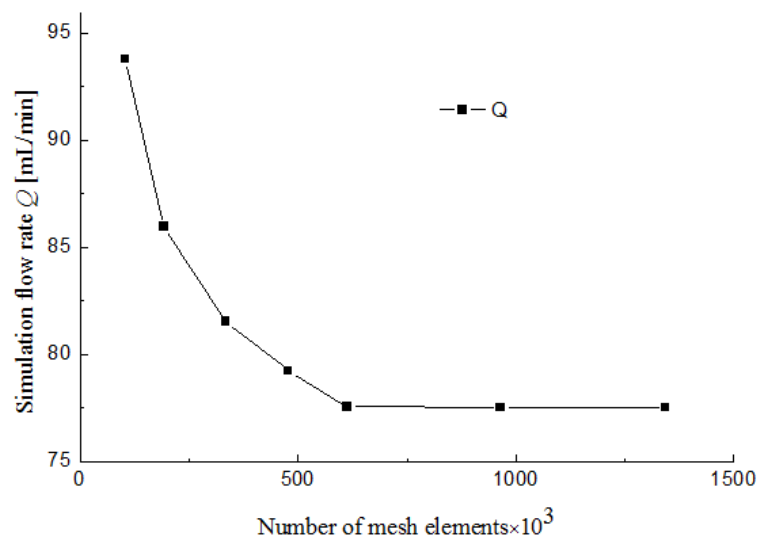

Fig. 3. Curves of simulation flow rate with different number of mesh elements

\subsection{Turbulence Model Comparison}

When the fluid flows through the HSG, turbulence including momentum diffusion and convection, an instantaneous and rapid variation of pressure and flow velocity will be formed due to interactions between fluid and all semi-hemispheres. Analytical solutions to this complex interaction become impossible. A numerical analysis tool, FLUENT was popularly applied to solve these problems. Acceptable simulation results can be achieved using this software. Two turbulence models, standard $k-\varepsilon$ and $R N G k-\varepsilon$ built in FLUENT, were used to compare pump flow simulation effectiveness.
Flow field simulation was conducted using HSGs of $3 \times 1,3 \times 2,3 \times 3$, and $3 \times 4$ for both standard $k-\varepsilon$ and RNG $k-\varepsilon$ models. Row and column intervals were kept constant at $S=H=12 \mathrm{~mm}$ in this simulation. Comparisons of simulation results and experimental data are listed in Table 2. Results indicated that the standard $k-\varepsilon$ and the $R N G \quad k-\varepsilon$ models had similar trends; but the $R N G \quad k-\varepsilon$ model had less error compared to the experimental results. Thus, the $R N G$ $k-\varepsilon$ turbulence model was used in the rest of this study. The variation of velocity, pressure, resistance coefficient and simulation flow rate were obtained from simultaneous solutions of continuity equation of numerical analysis, $\mathrm{N}-\mathrm{S}$ equations, and an $\mathrm{RNG} k-\varepsilon$ turbulence model in FLUENT.

Table 2 Comparison of measured flow rates and simulated using the standard $k-\varepsilon$ and the $R N G \mathrm{k}-\varepsilon$ turbulence models

\begin{tabular}{ccccc}
\hline \multirow{2}{*}{$\mathrm{RN}$ or $\mathrm{CN}$} & $\begin{array}{c}\text { Measured flow } \\
\text { rate* } \\
{[\mathrm{mL} / \mathrm{min}]}\end{array}$ & $\begin{array}{c}\text { Standard } \\
k-\varepsilon \text { model } \\
{[\mathrm{mL} / \mathrm{min}]}\end{array}$ & $\begin{array}{c}\mathrm{RNG} k-\varepsilon \text { model } \\
{[\mathrm{mL} / \mathrm{min}]}\end{array}$ \\
\hline \multirow{3}{*}{$\mathrm{RN}=3$} & $\mathrm{CN}=1$ & $39.4(0.642 \#)$ & 85.41 & 70.25 \\
\cline { 2 - 5 } $\mathrm{CN}=2$ & $47.9(0.733 \#)$ & 92.36 & 78.33 \\
\cline { 2 - 5 } $\mathrm{CN}=3$ & $51.1(0.827 \#)$ & 98.45 & 80.02 \\
\cline { 2 - 5 } $\mathrm{CN}=4$ & $52.5(0.743 \#)$ & 104.68 & 81.55 \\
\hline
\end{tabular}

Notes: row number (RN); column number (CN);

* Mean value of ten replications; \# Standard deviation

\subsection{Flow Field and Flow Rate Analysis with Different Row, Column Number of HSG}

\subsubsection{Analysis of Velocity Field}

Seven models for simulating pump flow fields were established and analysed. These seven models included pumps with $1 \times 3,2 \times 3,3 \times 3,4 \times 3,3 \times 1,3 \times 2$, and $3 \times 4$ HSGs. The velocity vector contours of the pump flow fields with HSGs $2 \times 3,3 \times 2,4 \times 3$, and $3 \times 4$ were selected as shown in Fig. 2. It can be seen that HSGs have different row and column numbers, with constant row and column intervals $(S=H=12 \mathrm{~mm})$.

The velocity fields of forward and reverse flow are shown in Fig. 4. The forward flows in Figs. 4a, c, e, g demonstrate that the fluid flows are blocked by the spherical surfaces of HSs, and the flow field are disturbed and formed a turbulent flow that increases the flow resistance. The flow velocity near the onequarter spherical region reduces. Meanwhile, part of the water flows through the gaps between HSs and forms some small flow streams and vortexes, which cause the flow velocity to reduce in those gaps, while most of the surrounding fluid flows through those 

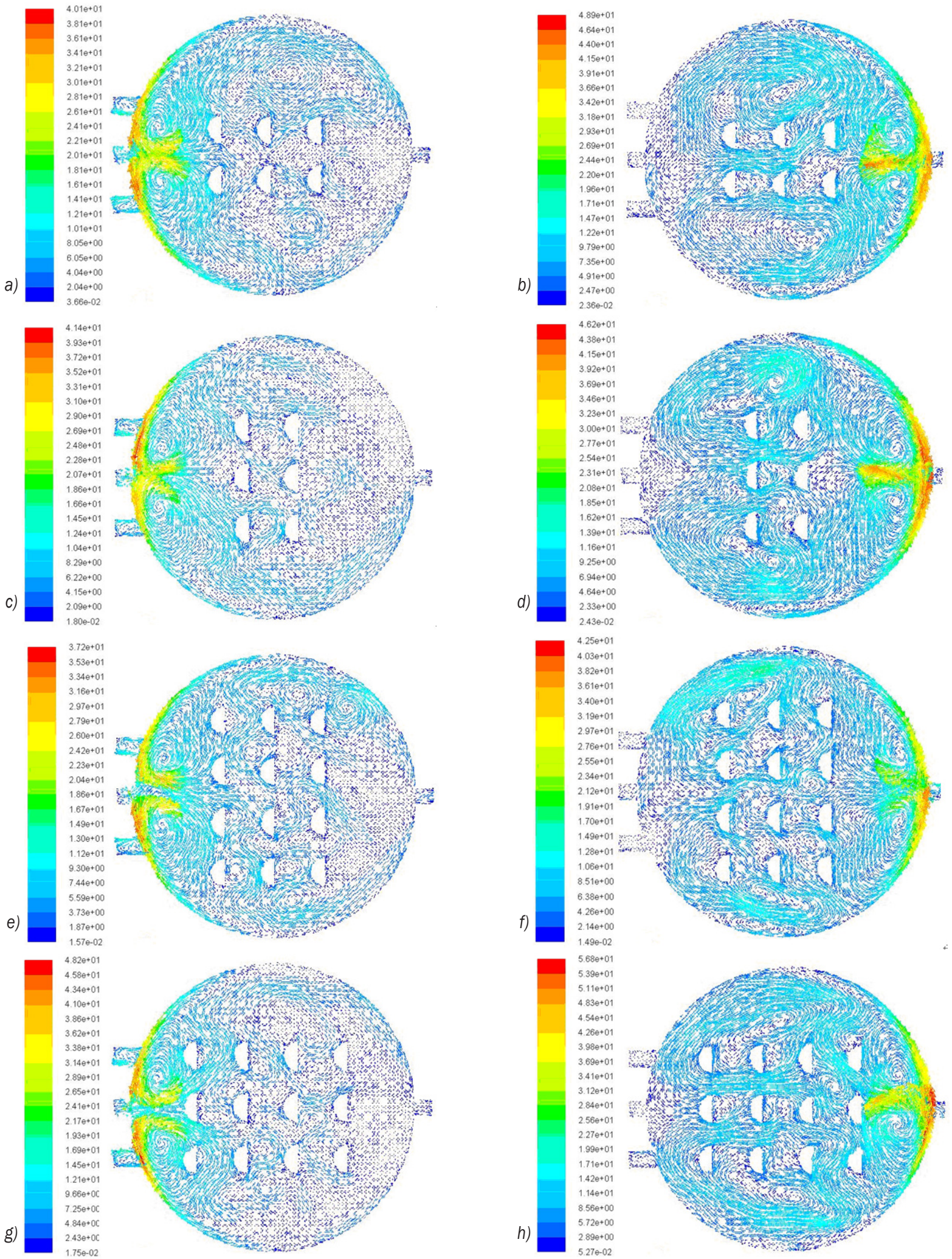

Fig. 4. Variation of velocity vectors along with the variations of row and column number in HSGs; a) HSG $2 \times 3$ in forward flow, b) HSG $2 \times 3$ in reverse flow; c) HSG $3 \times 2$ in forward flow, d) HSG $3 \times 2$ in reverse flow; e) HSG $4 \times 3$ in forward flow, f) HSG $4 \times 3$ in reverse flow, g) HSG 3×4 in forward flow, h) HSG $3 \times 4$ in reverse flow 
hemisphere-segments and the flow speed increases gradually. The reverse flows in Figs. $4 \mathrm{~b}, \mathrm{~d}, \mathrm{f}, \mathrm{h}$ demonstrate that fluid flow can be stopped by the flat semi-circular surfaces of HSs; and the water level surges that creates flow resistance sharply increase and the flow velocity rapidly drops. At the same time, part of the lagged fluid is pushed into the gaps and formed stronger streams and larger vortexes. These vortexes circulate within the HSG and produce increased flow resistance. Increasing flow resistance results in further consumption of the fluid kinetic energy, which greatly reduces fluid velocity. In summary, in the reverse flow the HSG consumes more fluid kinetic energy than in forward flow. It indicates that the reverse-flow resistance is dramatically bigger than forward flow resistance, which further explains the essential reason that HSG can play the role of a no-moving-part valve.

\subsubsection{Pressure and Resistance Coefficient Analysis}

When the fluid flows around the HSG, the boundary layer will split into many vortexes and produce velocity fluctuations that result in the changes of flow resistance and pressure in front and back of the HSG.

In order to obtain the changes of flow resistance and pressure in front and back of the HSG, as shown in Fig. 5, planes a-a, b-b, which are respectively 10 $\mathrm{mm}$ from the spherical surface of the left column hemisphere-segments and the flat semi-circular surface of the right column hemisphere-segments in HSG, are taken as reference planes. The intersection points between the center line of each HS row and the reference planes are respectively defined as $P_{i}, P_{i}^{\prime}(i=1, \ldots, m)$, where $m$ is the number of rows. Those points at the spherical surface side are expressed as $P_{i}$, while the points at the other side are written as $P_{i}{ }^{\prime}$. The average pressures on reference planes a-a and $\mathrm{b}-\mathrm{b}$ are defined as $\bar{P}$ and $\overline{P^{\prime}}$ respectively, and they are successively extracted from the corresponding flow field in Fig. 2. Hence, the pressure differences of the forward and reverse flowing around HSG are $\Delta p=\bar{P}-\overline{P^{\prime}}, \quad \Delta p^{\prime}=\overline{P^{\prime}}-\bar{P}, \quad$ respectively. The corresponding forward and reverse resistance coefficients can be calculated using Eqs. (1) and (2). The calculated results are shown in Table 3.

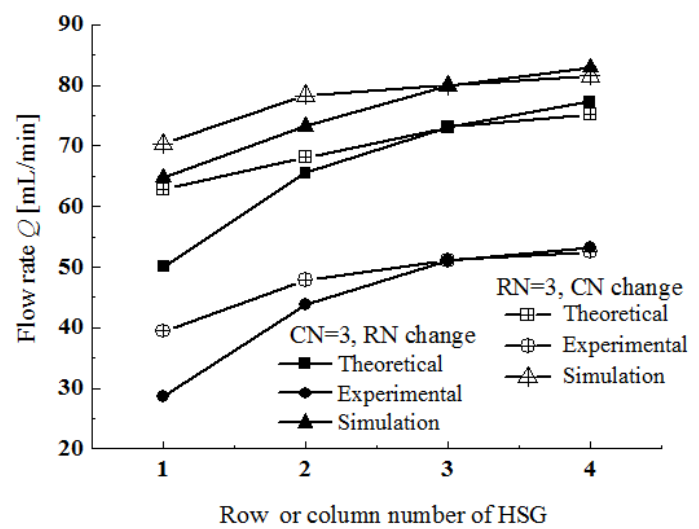

Fig. 5. Pressure points distribution of specified plane related to HSG

The results in Table 3 indicate that the pressure differences and resistance coefficients of forward and reverse flow around HSG increase along with the increasing of row number and column number. Moreover, the increase of pressure difference and resistance coefficient in the reverse flow is significantly greater than in forward flow.

\subsubsection{Simulation Analysis of Pump Flow Rate}

Based on the above simulation, the outlet flow rate was calculated. Fig. 6 shows the variation curves of the simulated flow rate when varying the flow resistance difference and row and column numbers of HSG. Through comparison and analysis, it can be found that the flow rate increases along with the increase of flow resistance difference, row and column numbers.

Table 3. Comparison of resistance coefficients for HSG with different array

\begin{tabular}{|c|c|c|c|c|c|c|c|c|c|}
\hline $\begin{array}{c}\text { Row number } \\
\text { change }\end{array}$ & $\begin{array}{c}\text { Flow } \\
\text { direction }\end{array}$ & $\mathrm{PD}_{\Delta p} /(\mathrm{pa})$ & $\mathrm{RC}$ & FRD & $\begin{array}{c}\text { Column number } \\
\text { change }\end{array}$ & $\begin{array}{c}\text { Flow } \\
\text { direction }\end{array}$ & $\mathrm{PD}_{\Delta p} /(p a)$ & $\mathrm{RC}$ & FRD \\
\hline \multirow{2}{*}{$1 \times 3$} & forward & 121960 & 3.072 & \multirow{2}{*}{0.8442} & \multirow{2}{*}{$3 \times 1$} & forward & 207912 & 3.5952 & \multirow{2}{*}{1.2239} \\
\hline & reverse & 254387 & 3.9163 & & & reverse & 224383 & 4.8191 & \\
\hline \multirow{2}{*}{$2 \times 3$} & forward & 175525 & 3.506 & \multirow{2}{*}{1.259} & \multirow{2}{*}{$3 \times 2$} & forward & 235749 & 3.8934 & \multirow{2}{*}{1.4291} \\
\hline & reverse & 311647 & 4.765 & & & reverse & 263553 & 5.3225 & \\
\hline \multirow{2}{*}{$3 \times 3$} & forward & 249761 & 4.172 & \multirow{2}{*}{1.6388} & \multirow{2}{*}{$3 \times 3$} & forward & 267934 & 4.172 & \multirow{2}{*}{1.6378} \\
\hline & reverse & 413443 & 5.8108 & & & reverse & 305365 & 5.8098 & \\
\hline \multirow{2}{*}{$4 \times 3$} & forward & 316005 & 4.643 & \multirow{2}{*}{1.9103} & \multirow{2}{*}{$3 \times 4$} & forward & 326085 & 4.827 & \multirow{2}{*}{1.906} \\
\hline & reverse & 512817 & 6.556 & & & reverse & 368279 & 6.733 & \\
\hline
\end{tabular}

Remarks: pressure difference (PD); flow resistance difference (FRD); resistance coefficient (RC) 


\subsection{Influence Analysis of Row and Column Interval on Flow Field and Flow Rate}

The hemisphere-segment array group of $3 \times 4$ is used as an example, and the fixed row interval $S$ set at 8 $\mathrm{mm}$, and column intervals $H$ at $8 \mathrm{~mm}, 10 \mathrm{~mm}, 12 \mathrm{~mm}$, and $14 \mathrm{~mm}$ respectively; fixed column interval $H=10$ $\mathrm{mm}$, and row interval $S=6 \mathrm{~mm}, 8 \mathrm{~mm}, 10 \mathrm{~mm}, 12 \mathrm{~mm}$ respectively. Thus, there were seven hemispheresegment groups with different intervals. Seven models of pump flow field with different HSGs were established and simulated. The simulated data are shown in Table 4.

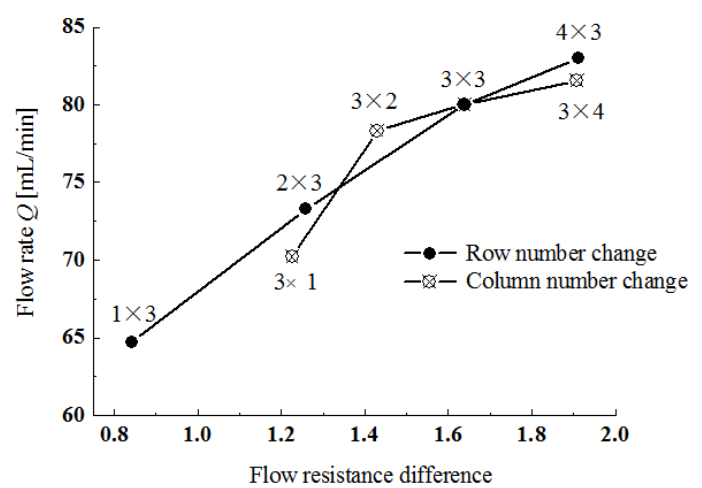

Fig. 6. Variation curves of simulation flow rate along with the variations of flow resistance, row and column number in HSG

From Table 4, it can be determined that the pressure differences, resistance coefficients, and simulation flow rates decrease specifically with the increasing of row interval; while increasing column interval the pressure differences, resistance coefficients and simulation flow rates increase. Through analysis, it can be known that the transverse interference and collision of fluid in the gaps of HSG will be weakened by increasing the row interval, and the flow in those gaps increase. Then the blocked fluid is evacuated. So, the fluid resistance is reduced, and the pressure loss and resistance coefficients is reduced. The increasing of column interval will weaken the covering flow effect of HSs in a longitudinal arrangement, and the other HSs behind the first column gradually leave the wake flow influence region of the first column hemisphere-segments, so the pressure difference and resistance coefficient gradually increase. The flow rate increases along with the increasing of flow resistance difference.

When the fluid flows through the region of HSG, turbulences and vortexes are generated that can helpfully mix the fluid with different components. The internal of dramatic turbulences and vortexes can be considered as a chaotic region. Because of multiple HSs arranged in transverse and longitudinal, the turbulences and vortexes generated by the fluid flowing through one or one row HSs have not yet spread out and then immediately are pushed into the influence region of next or next row HSs. In this way, multiple forms of chaos are generated. So, even if the "blind region" will be reduced or even disappear. Such turbulences and vortexes with overlays of layer upon layer can help fluids with different concentration,

Table 4. Variation comparison of pressure difference, resistance coefficient and pump flow rate along with row, column interval variations

\begin{tabular}{|c|c|c|c|c|c|c|}
\hline \multicolumn{2}{|c|}{ Interval [mm] } & Flow direction & $\mathrm{PD}_{\Delta p} /(p a)$ & FRC & FRD & Simulation flow rate $[\mathrm{mL} / \mathrm{min}]$ \\
\hline \multirow{8}{*}{$H=8$} & \multirow{2}{*}{$S=8$} & forward & 238992 & 4.927 & \multirow{2}{*}{1.834} & \multirow{2}{*}{82.731} \\
\hline & & reverse & 289521 & 6.761 & & \\
\hline & \multirow{2}{*}{$S=10$} & forward & 221196 & 4.862 & \multirow{2}{*}{1.7645} & \multirow{2}{*}{82.26} \\
\hline & & reverse & 270910 & 6.6265 & & \\
\hline & \multirow{2}{*}{$S=12$} & forward & 206504 & 4.6333 & \multirow{2}{*}{1.6132} & \multirow{2}{*}{81.019} \\
\hline & & reverse & 242708 & 6.2465 & & \\
\hline & \multirow{2}{*}{$S=14$} & forward & 200971 & 4.512 & \multirow{2}{*}{1.417} & \multirow{2}{*}{80.01} \\
\hline & & reverse & 239143 & 5.929 & & \\
\hline \multirow{8}{*}{$S=10$} & \multirow{2}{*}{$H=6$} & forward & 72821 & 4.935 & \multirow{2}{*}{1.673} & \multirow{2}{*}{80.25} \\
\hline & & reverse & 154381 & 6.608 & & \\
\hline & \multirow{2}{*}{$H=8$} & forward & 221196 & 4.862 & \multirow{2}{*}{1.7645} & \multirow{2}{*}{82.26} \\
\hline & & reverse & 270910 & 6.6265 & & \\
\hline & \multirow{2}{*}{$H=10$} & forward & 260452 & 4.846 & \multirow{2}{*}{1.819} & \multirow{2}{*}{82.48} \\
\hline & & reverse & 378207 & 6.665 & & \\
\hline & \multirow{2}{*}{$H=12$} & forward & 306045 & 4.9135 & \multirow{2}{*}{1.859} & \multirow{2}{*}{82.55} \\
\hline & & reverse & 482780 & 6.7725 & & \\
\hline
\end{tabular}

Remarks: pressure difference (PD); flow resistance difference (FRD); flow resistance coefficient (FRC) 
density and viscosity to mix in a highly uniform manner.

\section{EXPERIMENTAL VERIFICATION}

\subsection{Devices and Methods for Pump Flow Rate Test}

The test of the pump flow rate was carried out at zero pressure difference between inlet and outlet. The pump flow rate test principle diagram is shown in Fig. 7. The water level in the reservoir (a measuring cup) was kept at the same height as the water level in three inlet tubes and one outlet tube. The signal generator and power amplifier provide the driving voltage and frequency to drive the prototype pump. The weight of the liquid flowing out of the pump chamber in a fixed time interval was measured with an electronic balance, which had a resolution of $0.01 \mathrm{~g}$. The flow rate was repeatedly measured, and the average value was then calculated. Pure water was used as the pump fluid. The driving voltage and frequency were $160 \mathrm{~V}$ and $6 \mathrm{~Hz}$, respectively. Pump flow rates were successively measured under different hemisphere-segment array groups with the following combinations of row and column numbers (row by column): $1 \times 3,2 \times 3,3 \times 3$, $4 \times 3,3 \times 1,3 \times 2,3 \times 3,3 \times 4$. Tests were also conducted for seven hemisphere-segment array groups $3 \times 4$ with different rows and column intervals (for fixed column interval $H=8$, row interval $S=8,10,12,14$; fixed row interval $S=10$, column interval $H=6,8,10,12$ ).

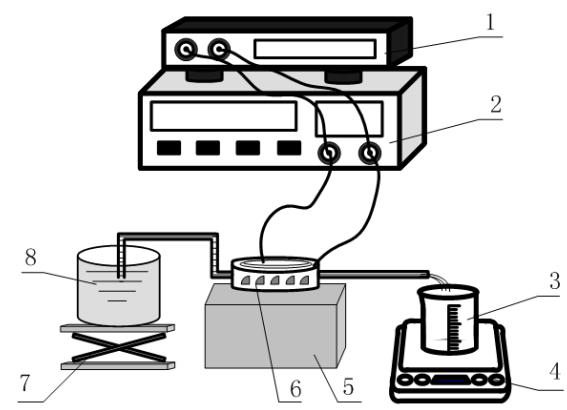

Fig. 7. Pump flow rate test principle diagram 1. power amplifier; 2. signal generator; 3. graduated glass; 4. electronic balance; 5. cushion block; 6. prototype pump 7. heightadjustable table; 8. reservoir

\subsection{Comparisons of Pump Flow Rates}

The theoretical pumping flow rate was calculated according to Eq. (7). The diameter of the piezoelectric vibrator is $50 \mathrm{~mm}$. When the frequency $f$ is $6 \mathrm{~Hz}$ the amplitude of the vibrator measured by CCD micro displacement sensor is $1.512 \mathrm{~mm}$. The forward and reverse resistance coefficients are taken from Tables 3 and 4. By calculating and analysing the theoretical flow rates, experimental flow rates, and simulation flow rates are shown in Figs. 8 and 9. All experiments were conducted under the same conditions and repeated 10 times. The mean values of flow rate and standard deviations are shown in Tables 5 and 6.

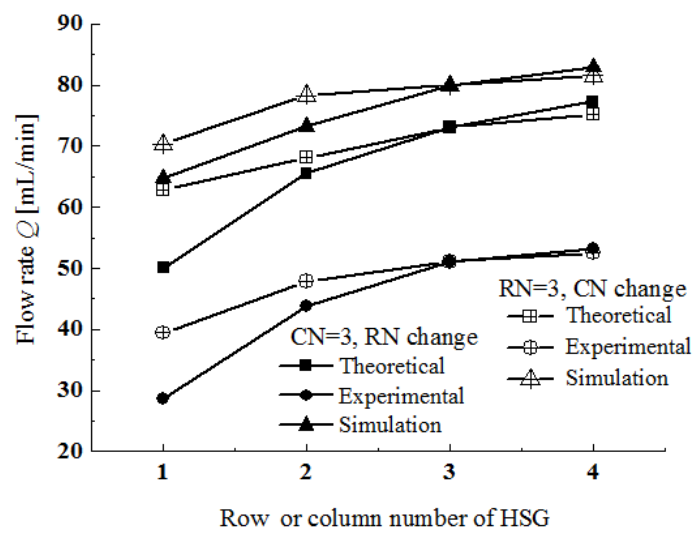

Fig. 8. Curves of pump flow rate with different $C N$, RN of HSGs; column number (CN), row number (RN)

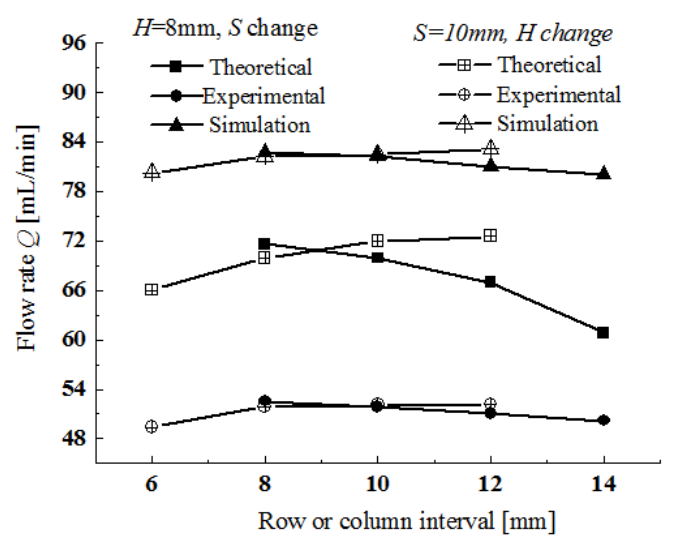

Fig. 9. Curves of pump flow rate with different $S, H$ of HSGs; column interval $(H)$, row interval $(\mathrm{S})$

Fig. 8 shows the curves of pump flow rates with different row and column numbers of HSGs. These curves indicate that theoretical, experimental and simulated flow rates all increase along with the increase of row or column number, and they have the same trend. The average deviations of simulation, theoretical flow rates relative to the experimental flow rates with different row number are $42.0 \%$ and 34.3 $\%$, respectively. The average deviations of simulation, theoretical relative to experimental flow rates with different column number are respectively $39.0 \%$ and $31.8 \%$. 
Table 5. Mean value and standard deviation of ten repeated experimental results in Fig. 8

\begin{tabular}{cccc}
\hline \multicolumn{2}{c}{ RN or CN } & \multicolumn{1}{c}{$\begin{array}{c}\text { Mean value of } \\
\text { flow rate }[\mathrm{mL} / \mathrm{min}]\end{array}$} & Standard deviation \\
\hline \multirow{3}{*}{$\mathrm{CN}=3$} & $\mathrm{RN}=1$ & 28.6 & 0.8310 \\
\cline { 2 - 4 } & $\mathrm{RN}=2$ & 43.9 & 0.7222 \\
\cline { 2 - 4 } & $\mathrm{RN}=3$ & 51.1 & 0.8267 \\
\cline { 2 - 4 } & $\mathrm{RN}=4$ & 53.2 & 0.7481 \\
\hline \multirow{3}{*}{$\mathrm{RN}=3$} & $\mathrm{CN}=1$ & 39.4 & 0.6422 \\
\cline { 2 - 4 } & $\mathrm{CN}=2$ & 47.9 & 0.7325 \\
\cline { 2 - 4 } & $\mathrm{CN}=3$ & 51.1 & 0.8267 \\
\cline { 2 - 4 } & $\mathrm{CN}=4$ & 52.5 & 0.7428 \\
\hline
\end{tabular}

Notes: row number (RN), column number (CN)

Table 6. Mean value and standard deviation of ten repeated experimental results in Fig. 9

\begin{tabular}{cccc}
\hline \multicolumn{2}{c}{ Interval $[\mathrm{mm}]$} & $\begin{array}{c}\text { Mean value of flow } \\
\text { rate }[\mathrm{mL} / \mathrm{min}]\end{array}$ & Standard deviation \\
\hline \multirow{3}{*}{$\mathrm{H}=8$} & $\mathrm{~S}=8$ & 52.6 & 0.6962 \\
\cline { 2 - 4 } & $\mathrm{S}=10$ & 51.9 & 0.7214 \\
\cline { 2 - 4 } & $\mathrm{S}=12$ & 51.08 & 0.7229 \\
\cline { 2 - 4 } & $\mathrm{S}=14$ & 50.2 & 0.7871 \\
\hline \multirow{3}{*}{$\mathrm{S}=10$} & $\mathrm{H}=6$ & 49.4 & 0.8690 \\
\cline { 2 - 4 } & $\mathrm{H}=8$ & 51.9 & 0.8143 \\
\cline { 2 - 4 } & $\mathrm{H}=10$ & 52.1 & 0.8022 \\
\cline { 2 - 4 } & $\mathrm{H}=12$ & 52.2 & 0.6465 \\
\hline
\end{tabular}

Fig. 9 shows pump flow rate variation curves with different row and column intervals. It can be seen that the simulation, theoretical, and experimental flow rates all decrease along with the increase of row interval, and all increase along with the increase of column interval; the pump flow rates change slowly in the whole variation range of row and column interval. The average deviations of simulation, theoretical flow rates relative to experimental flow rates with different row interval are $37 \%$ and $23.4 \%$, respectively. The average deviations of simulation, theoretical flow rates relative to experimental flow rates with different column interval are, respectively, $37.2 \%$ and $27 \%$.

Comprehensively analysing Table 3 and Fig. 8, as well as Table 4 and Fig. 9, it can be shown that the simulation, theoretical, and experimental flow rates have the same variation trends. Moreover, all of the flow rates increase along with the increasing of flow resistance difference. When the change of flow resistance difference is small, the change of corresponding pump flow rate is al small. The simulation results had the same trend with measured ones. This could provide a guideline for pump designers and researchers. It is also valuable for other researchers in attempting improve their work in the future. Some researchers in reference [5] indicated that the relative error between theoretical and experimental results could be as high as $90 \%$; but the same trend could be used to verify the concept of the design. For the deviations of simulation and theoretical flow rates relative to experimental flow rates in this research, the reasons can be concluded as the following:

(1) Theoretical flow rate expressions and simulation flow rate are derived and analysed according to the simplified model. Moreover, it is ignored that a large amount of heat energy is consumed due to shock waves, turbulence, and fluctuations in the actual transient flow field, so the mechanical energy is no longer conserved. This causes the theoretical and simulation flow rates bigger than the experimental flow rate.

(2) In experiments, boundary layers of the flow field separate and split into many vortexes after the fluid flowing around the hemisphere-segment group, which causes fluid energy loss, so the experimental flow rate is smaller than theoretical and simulation flow rates.

(3) In addition, the errors caused by the vortex circuitously flowing, colon, bubbles, pump sealing, experiment environment and other factors randomly occur, and cannot be quantified by theory or experiments, which directly affect the theoretical analysis and experimental results.

\section{CONCLUSIONS}

Through theoretical research, simulation analysis, and experimental verification, we have studied in depth the flow field and pumping performance of a valveless piezoelectric pump with HSG. The conclusions are as follows:

(1) The influence of the HSG's different row and column number on pumping performance is simulated and analysed. Moreover, the variation laws of velocity, pressure difference, and resistance coefficient along with the variations of row and column number are obtained. The increasing of row and column number directly increases flow resistance difference of HSG. The pump flow rate increases with the increasing of flow resistance difference. At the same time, it is found that the pump flow rate is bigger by increasing the row number than by increasing the column number, and the pumping performance is better improved by increasing the row number than by increasing the column number.

(2) The influence of the HSG's different row and column intervals on pumping performance is simulated and analysed. Moreover, the variation 
laws of velocity, pressure difference, flow rate and resistance coefficient along with the variations of row and column intervals are obtained. It is found that the influence of interval change on flow resistance difference and pump flow rate is not significant, but the interval changes have a large influence on the size and strength of vortexes in flow field. The flow field will generate more and stronger vortexes by increasing the row and column intervals of HSG and the fluid can be well blended. Thus, the function of mixing fluid for a valveless piezoelectric pump with HSG can be well realized.

(3) The pumping performance experiments were carried out with different row and column numbers, and different row and column intervals of HSGs. The variation trend of simulation, theoretical and experimental flow rates was the same, and the reason for the deviations was explained. Finally, simulation results were verified through pump flow rate experiments.

\section{ACKNOWLEDGMENTS}

This project was supported by National Natural Science Foundation of China (No.51375227, 51475221), Zhejiang Provincial Natural Science Foundation of China (No. LY13E050015), and Application Type University Construction Project of Qingdao Agricultural University.

\section{REFERENCES}

[1] Izzo, I., Accoto, D., Menciassi, A., Schmitt, L., Dario, P. (2007). Modelling and experimental validation of a piezoelectric micropump with novel no-moving-part valves. Sensors and Actuators A: Physical, vol. 133, no. 1, p. 128-140, DOl:10.1016/j.sna.2006.01.049.

[2] Zhang, J.H., Li, Y.L., Xia, Q.X. (2007). Analysis of the pump volume flow rate and tube property of the piezoelectric valveless pump with Y-shape tubes. Chinese Journal of Mechanical Engineering, vol. 43, no. 11, p. 136-141, D0l:10.3901/ JME.2007.11.136. (in Chinese)

[3] Zhang, J.H., Lu, J.ZH., Xia, Q.X., Kou, J., Ren, G. (2008). Working principle and characteristics of valve-less piezoelectric pump with Y-shape tubes for transporting cells and macromolecule. Chinese Journal of Mechanical Engineering, vol. 44, no. 9, p. 92-99, DOI:10.3901/JME.2008.09.092. (in Chinese)

[4] Morris, C.J., Forster, F.K. (2003). Low-order modelling of resonance for fixed-valve micropumps based on first principles. Journal of Microelectromechanical Systems, vol. 12, no. 3, p. 325-334, D0I:10.1109/JMEMS.2003.809965.
[5] Huang, Y., Zhang, J.H., Hu, X.Q., Xia, Q.X., Huang, W.Q., Zhao, C.S. (2010). Dynamics analysis and experiment on the fishtailing type of valveless piezoelectric pump with rectangular vibrator. Science China Technological Sciences, vol. 53, no. 12, p. 3241-3247, Dol:10.1007/s11431-010-41760.

[6] Huang, J., Zhang, J.H., Xun, X.C., Wang, S.Y. (2013). Theory and experimental verification on valveless piezoelectric pump with multistage Y-shape treelike bifurcate tubes. Chinese Journal of Mechanical Engineering, vol. 26, no. 3, p. 462-468, DOI:10.3901/CJME.2013.03.462.

[7] Huang, J., Zhang, J.H., Wang, S.Y., Liu, W.D. (2014). Analysis of the flow rate characteristics of valveless piezoelectric pump with fractal-like Y-shape branching tubers. Chinese Journal of Mechanical Engineering, vol. 27, no. 3, p. 628-634, DOI:10.3901/CJME.2014.03.628.

[8] Stemme, E., Stemme, G. (1993). A valve-less diffuser/nozzlebased fluid pump. Sensors and Actuators A: Physical, vol. 39, no. 12, p. 159-167, D0l:10.1016/0924-4247(93)80213-Z.

[9] Nguyen, N-T., Huang, X. (2000). Numerical simulation of pulsewidth-modulated micropumps with diffuser/nozzle elements. Technical Proceedings of the International Conference on Modeling and Simulation of Microsystems, p. 636-639.

[10] Rife, J.C., Bell, M.I. Horwitz, J.S., Kabler, M.N., Auyeung, R.C.Y., Kim, W.J. (2000). Miniature valveless ultrasonic pumps and mixers. Sensors and Actuators A: Physical, no. 86, no. 1-2, p. 135-140, DOI:10.1016/S0924-4247(00)00433-7.

[11] Sheen, H.J., Hsu, C.J., Wu, T.H., Chu, H.C., Chang, C.C., Lei, U. (2008). Experimental study of flow characteristics and mixing performance in a PZT self-pumping micromixer. Sensors and Actuators A: Physical, no. 139, no. 1-2, p. 237-244, DOI:10.1016/j.sna.2007.02.031.

[12] Xia, Q.X., Zhang, J.H., Lei H, Cheng W. (2009). Theoretical analysis and experimental verification on flow field of piezoelectric pump with unsymmetrical slopes element. Chinese Journal of Mechanical Engineering, vol. 22, no. 5, p. 735-744, DOI:10.3901/CJME.2009.05.735. (in Chinese)

[13] Ji, J., Zhang, J.H., Xia, Q.X., Wang, S.Y., Huang, J., Zhao, C.S. (2014). Theoretical analysis and experimental verification on valve-less piezoelectric pump with hemisphere-segment bluffbody. Chinese Journal of Mechanical Engineering. vol. 27, no. 3, p. 595-605, D0I:10.3901/CJME.2014.03.595.

[14] Singhal, V., Garimella, S.V., Murthy, J.Y. (2004). Low Reynolds number flow through nozzle-diffuser elements in valveless micropumps. Sensors and Actuators A: Physical, vol. 113, no. 2, p. 226-235, DOI:10.3901/CJME.2014.03.595.

[15] Zhang, J.H., Wang, S.Y., Yang, D.P. (1999). A study on a valveless, piezoelectric diffuser/nozzle-based fluid pump: analysis of vibration and the changed amount of the chamber volume. Piezoelectric \& Acoustooptics, vol. 21, no. 6, pp. 459460 (in Chinese).

[16] Zhang, J.H., Wang, S.Y. (2001). Study of piezoelectric valveless diffuser/nozzle-based fluid pump: one-way flow principle and the pump flow. Piezoelectric \& Acoustooptics, vol. 23, no. 1, p. 23-25. (in Chinese) 